\title{
Opportunités du téléphone portable pour la construction de nouvelles représentations
}

Opportunities of the mobile phone for the construction of new représentations Oportunidades del teléfono móvil para la construcción de nuevas representaciones

\section{Natacha Cyrulnik}

\section{OpenEdition}

\section{Journals}

Édition électronique

URL : http://journals.openedition.org/ctd/595

DOI : $10.4000 /$ ctd. 595

ISSN : 2491-1437

Éditeur

Chaire Unesco Pratiques émergentes en technologies et communication pour le développement

Référence électronique

Natacha Cyrulnik, «Opportunités du téléphone portable pour la construction de nouvelles représentations », Communication, technologies et développement [En ligne], 6 | 2018, mis en ligne le 18 décembre 2018, consulté le 02 mai 2019. URL : http://journals.openedition.org/ctd/595 ; DOI $10.4000 /$ ctd. 595

Ce document a été généré automatiquement le 2 mai 2019.

Communication, technologies et développement 


\title{
Opportunités du téléphone portable pour la construction de nouvelles représentations
}

\author{
Opportunities of the mobile phone for the construction of new représentations \\ Oportunidades del teléfono móvil para la construcción de nuevas \\ representaciones
}

Natacha Cyrulnik

Dans le cadre d'ateliers audiovisuels, Pilar Arcila et Jean-Marc Lamoure ont proposé à des jeunes des quartiers défavorisés de la ville de Port-Saint-Louis-du-Rhône de faire de petits films avec leurs téléphones portables. Ces réalisateurs font de nombreux ateliers en région PACA depuis des années, ce qui leur permet d'avoir une appréhension de ces situations en constante évolution. Même s'ils affirment devoir toujours inventer une nouvelle manière d'aborder un atelier, leur expérience permet d'analyser le cas particulier du téléphone portable afin de mesurer ce qui constitue un nouvel enjeu dans le cadre d'atelier d'éducation artistique au cinéma (Cyrulnik, 2016), et de mieux en cerner les apports.

2 Ces ateliers d'éducation artistique au cinéma permettent à la fois de favoriser un esprit critique vis-à-vis des images si facilement consommées, en même temps qu'ils offrent aux participants l'occasion d'entrer en action pour définir ce qu'ils ont à raconter, de composer un récit, et ainsi de s'affirmer. Pourtant cette nécessité de savoir lire avec du recul des images s'apparente souvent plus à un devoir civique qu'à l'appréhension d'une véritable dimension artistique qui favoriserait un épanouissement personnel et qui serait prioritaire.

«Il appartient à celui qui délivre [l'éducation aux médias] de construire en même temps la motivation de l'apprenant, et la conviction qu'il a raison de s'éduquer ainsi ; [...] que [cette éducation] vise de manière primordiale à développer la prise de distance des apprenants avec les médias eux-mêmes pour que l'enseignement ne sombre pas dans le bric-à-brac ou le n'importe quoi. La construction de l'esprit critique vis-à-vis de la spécialité constitue un objectif majeur » (Porcher, 2006 : 80). 
Aux propos de Louis Porcher sur l'éducation aux médias s'ajoutent ceux de Serge Tisseron :

«Il est urgent de réintroduire la possibilité pour chacun de s'approprier ses expériences d'images avec ses propres moyens. Pour cela, l'éducation aux médias doit créer des espaces d'échanges verbaux autour des images, mais aussi des espaces où les enfants sont invités à créer leurs propres images, à les transformer et à les manipuler, et enfin des espaces où ils peuvent être sollicités pour les mettre en scène en groupe de manière ludique et créative » (Tisseron, 2002 : 140).

4 L'engagement de la personne qui participe à des ateliers d'éducation artistique au cinéma est primordial dès le départ, et doit être prolongé notamment par le jeu et la création. Différentes manières d'aborder la pédagogie au cinéma sont pensées en fonction aussi des intentions de chacun, participants comme porteurs de ces ateliers (Cyrulnik, 2016). Et le téléphone portable comme outil à priori accessible offre justement une nouvelle situation d'expérimentation des images à construire.

Pour ce qui concerne Pilar Arcila et Jean-Marc Lamoure, ils ont toujours attaché de l'importance à savoir capter des images et des sons sur un lieu particulier afin d'en extraire un récit par la suite au montage. Ils cherchent ainsi à rendre compte d'une " sensation collective » à partir de ces images et de ces sons récoltés par les participants. S'ils notent déjà dans leurs différents ateliers une sorte d'appétence de la part des jeunes pour l'aspect technique qui pourrait venir de leur utilisation quotidienne des écrans (Cyrulnik, 2016 : 89), en quoi le fait d'utiliser des téléphones portables pour mener à bien ces ateliers changent-ils leur pratique et surtout l'expérience qu'ils vont en faire? En quoi le téléphone portable change-t-il l'expérience artistique qui va être faite de ces territoires particuliers ? Et, finalement, la représentation filmique qui va être réalisée de ces espaces souvent stigmatisés à l'aide d'un téléphone portable va-t-elle permettre d'en changer les représentations sociales (Moscovici, 1984)?

6 Le téléphone portable évoque pour les jeunes un lien avec le monde à travers son utilisation en tant que téléphone ou à travers les réseaux sociaux; il suggère aussi des contacts, photos ou vidéos dans un registre plus intime. Partir de quelque chose d'intime qui devient universel, ou en tout cas qui résonne pour les autres est le fondement de la dimension artistique (Meirieu, 2003; Guénoun, 2006; Menger, 2002). Dans le processus même de création, un positionnement par rapport aux autres s'opère. Ces situations d'ateliers d'éducation artistique au cinéma offrent une manière d'essayer de s'exprimer, voire de se faire entendre. Le téléphone portable exacerberait cela, c'est notre hypothèse. Cette dualité ou cette complémentarité entre un registre intime et une connexion au monde plus universelle peut être porteuse pour créer justement de nouvelles représentations du monde.

7 Ainsi les différents niveaux d'analyse d'une méthode pédagogique pour ce genre d'atelier vont permettre de mesurer en quoi un tel dispositif change dans la mesure où il fait appel aux téléphones portables des participants. Cet objet technique va ainsi entrer dans un registre de plus en plus intime afin de construire une représentation filmique dans un premier temps. D'autres niveaux de représentations entrent en jeu à leur tour en cherchant à mieux comprendre la situation dans ces territoires particuliers afin d'en changer cette fois les représentations sociales et ce qu'elles impliquent pour les habitants. Ces représentations dans une esthétique relationnelle et une approche plus rhétorique aident ainsi les jeunes à mieux se positionner face à cet objet technique pour prendre place dans le monde. D'un objet technique qui permet plusieurs niveaux de 
représentations, nous en viendrons petit à petit à des considérations intimes et politiques à la fois.

\section{Les différents niveaux d'analyse d'une méthode}

\section{Le dispositif des ateliers d'éducation artistique au cinéma avec des téléphones}

En utilisant directement le téléphone portable des participants à l'atelier pour réaliser un film dans le cadre d'atelier d'éducation artistique au cinéma, un processus de création particulier se met en place. Si les situations de pédagogies au cinéma peuvent être listées (Cyrulnik, 2016), il est toujours nécessaire de l'adapter à la réalité de chaque nouvel atelier à mettre en œuvre. Il n'y a pas une formule magique à appliquer, juste quelques points communs ou divergents à pointer pour s'en emparer en fonction de chaque situation. Dans le cas de l'utilisation des téléphones portables, ce n'est plus le porteur de projet qui arrive avec sa caméra, qui lui conférait déjà un certain statut et qui devenait une première entrée en matière pour établir un contact. C'est plutôt son expérience en tant que professionnel qui importe, son statut! Cela ne se voit pas de la même manière que j'ai pu personnellement l'expérimenter durant plus de dix ans dans de nombreuses cités en France par la présence forte de cet objet caméra qui est marquante, mais par la seule personne qui vient accompagner un atelier. C'est-à-dire que le travail de rue que j'ai mené durant des années dans les cités ne peut pas se mettre en œuvre de la même manière, ne serait-ce que parce qu'il a lieu dans la rue et que l'objet caméra est si visible qu'il devient notre premier objet de discussion, que ce ne soit en évoquant la télévision ou les futurs spectateurs que cet objet suggère déjà ! Pour mener un atelier avec des téléphones portables, celui-ci doit donc être annoncé aux enfants des quartiers où les réalisateurs viennent afin que cela se sache à l'avance. Le statut des futurs accompagnants en tant que réalisateurs devient l'atout qui va faire que cet atelier peut avoir lieu! Ce statut professionnel annonce une forme d'autorité (Arendt, 1954) et de respect qui va être mise à profit de ce cadre pédagogique naissant.

Pourtant l'objet qui sert à filmer fédère par sa seule présence d'entrée de jeu, qu'il soit une caméra apportée ou le téléphone portable que chaque participant amène. Les mobiles ont un lien avec leur propriétaire dans la mesure où celui-ci y a déjà investi un morceau de sa vie : musiques, photos, échanges par réseaux sociaux, textos ou mails, etc. Et ils sont utilisables tout le temps: lors de l'atelier, mais en dehors aussi. Le temps de l'atelier s'étire ainsi. Le cadre est moins précis de ce point de vue, mais les sujets abordés durant la journée peuvent être prolongés de manière plus individuelle (voir plus intime) le soir. Cela ouvre d'autres possibilités créatives... Et cette question d'un autre rapport au temps est présente aussi dans le fait que les films que les jeunes vont réaliser seront sans doute plus courts, pour pouvoir aussi circuler sur les portables ensuite... Le téléphone suggère donc une intimité et un rapport au temps particulier.

Il suggère aussi un rapport à l'espace avant même qu'il soit utilisé pour filmer. L'atelier d'éducation artistique au cinéma offre des situations concrètes à filmer, encore plus quand il se passe dans des quartiers marginalisés. Les personnalités qui se présentent face à la caméra permettent de filmer des acteurs dans des décors marquants, encore plus sur ces terrains particuliers que sont ces quartiers (Authier J.Y., Bacqué M.H., et Guérin-Pace 
F., 2007)! Le lieu où l'on se trouve influence donc beaucoup la construction du film, ne serait-ce que parce que le sujet de l'environnement proche existe déjà dans les images !

11 Dans le cadre de ces ateliers que Jean-Marc et Pilar ont menés avec des téléphones portables, le premier constat était la présence nouvelle de toutes ces caméras potentielles: les jeunes ayant chacun leur téléphone portable, la déambulation sur le territoire les a obligés à se poser constamment la question de la place qu'ils choisissaient de prendre. Cette réflexion qui peut paraître existentielle et politique, influence aussi les jeunes pour faire attention les uns les autres tout en affirmant quelques premiers choix : je me place à cet endroit, pour voir cela de telle manière, etc. (Cyrulnik, 2016:90). Cette multiplicité des caméras impose une affirmation individuelle et collective à la fois. Il faut définir sa place sur le lieu en même temps qu'il faut prendre les autres en compte, qu'ils soient dans le champ de ce qui est filmé ou pas! La présence du groupe fait partie intégrante du projet dès le début.

\section{La construction de sens avec ces objets techniques et intimes à la fois}

12 Par ailleurs, toujours en termes de méthode, les téléphones portables imposent aussi le fait que le dérushage soit réalisé tous les soirs : il faut vider les cartes mémoires afin de pouvoir les réutiliser le lendemain pour la suite de l'atelier! Ainsi l'utilisation de téléphone portable, par ce dérushage quotidien, crée des situations qui suscitent une réflexion de préfiguration du montage chaque fin de journée... La technique impose une forme de réflexion, que ce soit pour anticiper le tournage du lendemain, comme pour se projeter dans le film dans sa globalité en train de se construire... (Cyrulnik, 2016: 89). C'est donc, à travers ces considérations qui peuvent sembler a priori très techniques, qu'une narration se met en place au rythme des impressions des participants. La technique se met bien au service d'une intention, qui elle-même se définit au fur et à mesure que l'on avance dans la découverte du cinéma... Un récit se construit principalement au montage, mais il témoigne toujours de ces découvertes, de ces expériences, dans un registre sensible. D'une approche intuitive au début, le matériau est de plus en plus analysé pour être mieux compris au final (Paille et Mucchielli, 2005).

13 C'est donc en discutant entre eux à partir des images et des sons qu'ils ont ramené chaque soir, qu'un film se construit. La technique audiovisuelle s'expérimente donc à partir du ressenti et des échanges. L'utilisation du téléphone portable offre la liberté d'avoir une caméra facile d'accès. Elle pose alors la question de l'importance à accorder aux considérations techniques et celles qui favorisent l'émancipation des participants (Rancière, 2008). L'objet associe alors la technique à la culture. C'est une forme de médiologie (Debray, 1994). "Dans médiologie, "médio" ne dit pas "média" ni "médium", mais "médiations", soit l'ensemble dynamique des procédures et corps intermédiaires qui s'interposent entre une production de signes et une production d'événements » (Debray, 1994 : 29). L'outil qui sert à filmer relie l'ensemble de ces éléments. Daniel Bougnoux insiste sur le fait que cette approche médiologique intervient «sur ces milieux, indissociablement sociaux et techniques, qui façonnent et recyclent nos représentations symboliques, et nous permettent de tenir ensemble » (1998: 67). Le téléphone portable pris en tant qu'objet caméra, mais en plus accessible, devient un outil qui favorise la mise en relation. Dans ce processus de médiologie (Debray, 1994 ; Bougnoux, 1998), l'outil téléphone permet à la fois de s'emparer d'un objet intime pour construire une nouvelle représentation filmique, du monde, et de soi. 

la plupart du temps du principe que c'est en les expérimentant par la pratique qu'elles vont être plus lisibles et compréhensibles. D'une pratique de terrain, des usages du téléphone portable et de la consommation des images au quotidien, des intentions de construction de représentations émergent. L'analyse de la méthode mise en place dans le cadre de ces ateliers de pratiques artistiques avec des téléphones, affirme donc des partispris pédagogiques, cinématographiques, éthiques et civiques à la fois.

\section{Les différents niveaux de représentations}

\section{Représentations filmiques, intimes et collectives}

Dans tous les cas, quel que soit l'outil pour filmer sur lequel s'appuie l'atelier, cet objet est perçu comme un prétexte pour aller vers les autres : ceux qui font l'atelier comme ceux qui sont filmés. Il permet d'amorcer un pas vers autrui. Il met aussi en position de réflexion, de recul, de distance, de déplacement, celui qui participe à cet atelier, et lui offre ainsi la possibilité de trouver sa place dans le monde... C'est ce que convoque le «re » de représentation : cette mise à distance et ce recul pour mieux comprendre une situation (Boungoux, 2006 : 53). C'est un temps de partage, des sentiments, des ressentis, qui sont mis en avant dans le cadre d'atelier. Il n'est pas question de formation dans ce cas, mais de sensibilisation. Cette distinction est très importante pour bien mesurer ce qui se joue... et appréhender le cinéma, ou l'art en général, de manière sensible (Cyrulnik, $2016: 92)$.

Les représentations qui se construisent sont alors d'ordre cinématographique : réaliser un film avec des images et des sons captés avec les téléphones, choisis et articulés entre eux. La représentation filmique est la première des représentations qui se construit. Dans notre monde

« médiaculturel » (définir où l'on se situe à ce croisement entre média et culture, selon Eric Maigret et Eric Macé, 2005), la consommation des images devient un premier appui pour se définir et envisager un film à réaliser. À cela s'associe le fait que des images (photos et vidéos intimes) et des sons (principalement des musiques sélectionnées) sont déjà dans le téléphone portable du participant. L'atelier de pratique artistique proposé va susciter la captation d'autres images et d'autres sons (même si celui-ci est souvent le parent pauvre techniquement dans ces outils). L'articulation entre des données intimes (qui vont être rendues forcément publiques cette fois afin de les mettre en commun pour réaliser un film ensemble) et une réflexion commune oblige le participant à se positionner collectivement et individuellement en même temps cette fois. Le téléphone portable peut aller dans sa famille le soir par exemple, ce que faisait très rarement la caméra auparavant. Des nouveaux territoires plus intimes sont alors rendus publics; le fait de filmer son chat ou sa sœur suggère un nouveau registre de communication. En plus du positionnement dans l'espace évoqué plus haut, le téléphone portable oblige à se définir dans entre les sphères publiques et privées, à en expérimenter les limites, à les mesurer, et à choisir sa place par rapport aux autres et au monde. La réflexion commence à devenir plus psychologique en même temps que politique. Ces questions, et les choix qu'elles suggèrent imposent de nouveaux niveaux de représentations.

Communication, technologies et développement, 6 | 2018 


\section{Représentations dans une esthétique relationnelle et rhétorique}

18 L'esthétique du film nous met en relation avec le monde dans une logique qui devient de plus en plus participative (Bourriaud, 2001). Ce sont les échanges entre tous les porteurs de téléphones qui vont composer le récit du film. À partir de ce qu'ils amènent chaque soir, une réflexion se développe pour construire un film. Quand Nicolas Bourriaud explique ce qu'est "l'esthétique relationnelle ", il témoigne de la dimension participative comme étant porteuse de sens tout en fédérant. Le "vivre ensemble » est évoqué ; il est même convoqué pour composer une œuvre, toute proportion gardée, qui va obliger à se positionner face à ces images et à ces sons afin de composer un récit et d'articuler une pensée (Paillé et Mucchielli, 2005). Il faut assumer des choix face aux autres téléphones, face aux autres, dans le monde ! L'importance de la relation est encore plus présente avec un téléphone qu'avec une caméra, techniquement et intimement. Elle exacerbe cela.

Pour autant, la caméra avec ce qu'elle représente en termes d'argent, de métiers (journalistes ou policiers), de confiance pour construire une relation quand on la prête, installe déjà des types d'interactions possibles. Le fait d'avoir un film à réaliser favorise donc les interactions. Jean-Marie Schaeffer (1999) justifiait déjà l'importance de la fiction (ou d'une narration en général) en disant qu'elle aide à «être-au-monde ». Le récit qui se compose, qu'il soit fictionnel ou documentaire dans le cadre de ces ateliers, favorise l'affirmation du positionnement du participant à plusieurs niveaux.

Mais, si l'esthétique est principalement relationnelle, le discours à l'intérieur du film est lié à celui sur le monde ; il est rhétorique nous dit Guillaume Soulez (2011). C'est aussi ce que les jeunes choisissent de garder ou de raconter d'eux-mêmes et de leur environnement qui est important. En construisant leur film, ils construisent l'image qu'ils souhaitent donner d'eux- mêmes et de leur territoire, qui lui-même est souvent stigmatisé. Les représentations sociales (Moscovici, 1984) peuvent s'en trouver aussi changées!

21 À partir la culture locale du quartier et de la proposition d'en construire une représentation, tout en prenant en compte une vision globale du monde ${ }^{1}$ (Appaduraï, 2001), les films réalisés dans le cadre de ces ateliers au cœur de ces quartiers témoignent au final d'une grande diversité culturelle. Le territoire filmé et raconté selon un type de discours filmique choisi témoigne toujours d'une manière ou d'une autre de tous les échanges au final. Chacun s'exprime individuellement à travers son film qu'il projettera aux yeux de tous. La représentation filmique de ce territoire particulier révèle une représentation de soi au sein de la société. La dimension politique de cet acte artistique s'affirme.

\section{L'expérimentation par l'art et l'engagement citoyen}

De l'apprentissage du langage cinématographique à l'expression de soi, la dimension artistique et la force de la création (Dewey, 1915 ; Meirieu, 2003 ; Menger, 2002 ; Guénoun, 2006) par la réalisation de ces films à l'aide des mobiles offrent aux participants l'occasion de s'affirmer. Le téléphone portable en tant qu'objet de création éloigne les jeunes de ces quartiers périphériques d'une fracture numérique dont ils pourraient souffrir, et des stigmates médiatiques dans lesquels ils se sentent souvent enfermés (Moscovici, 1984). D'une réflexion intime qui devient universelle puisqu'il y a acte de création, le film 
projeté à la fin révèle un point de vue et un parti-pris qui raconte beaucoup de ce que les participants ont choisi de révéler de leur vie. Les films d'ateliers laissent toujours un peu transparaître cela. La réalisation de films avec des mobiles participe ainsi à une forme de revendication d'une territorialité.

Le téléphone portable devient bien une nouvelle forme d'expression par le film, en expérimentant par l'art (Dewey, 1915). Mais si l'intimité de l'objet permet d'entrer dans différents registres de communication, ce sont les différents registres de représentations qui valorisent leur apport. Les enjeux des ateliers d'éducation artistique au cinéma sont toujours valables. Les mobiles favorisent une expérimentation de l'espace et de la place de l'autre qui en fait aussi un objet politique.

Une part d'engagement citoyen se développe dans la mesure où ces jeunes offrent leur propre regard sur le monde qui les entoure (Colleyn, 1993) en même temps qu'ils s'exposent aux yeux de tous lors de la projection publique qui clôture cette aventure cinématographique. Face à un grand besoin de reconnaissance (Honneth, 2010 ; Ricœur, 2004) de la part de ces jeunes, ces situations pédagogiques à fortiori avec des téléphones portables suscitent, par l'affirmation de toutes ces représentations (filmiques, territoriales, individuelles et collectives), une forme d'engagement citoyen qui aide à mieux vivre ensemble.

\section{BIBLIOGRAPHIE}

Arjun Appaduraï, Après le colonialisme - les conséquences culturelles de la globalisation, Barcelone, Petite Bibliothèque Payot/ Rivages, 2001.

Daniel Bougnoux, Introduction aux sciences de la communication, Paris, Éd. La Découverte, 1998.

Daniel Bougnoux, La crise de la représentation, Paris, Éd. La Découverte, 2006. Nicolas Bourriaud, L'esthétique relationnelle, Paris, Ed. Les presses du réel, 2001.

Denis Guénoun, Le théâtre est-il nécessaire ?, Paris, Penser le théâtre, Circé, 2006. Axel Honneth, La lutte pour la reconnaissance, Ed. Cerf, Coll. Passages, 2006.

Eric Maigret et Eric Macé, Penser les médiacultures - Nouvelles pratiques et nouvelles approches de la représentation du monde, Paris, Armand Colin/INA, 2005.

Guillaume Soulez, Quand le film nous parle, Rhétorique, Cinéma, télévision, PUF, Coll. Lignes d'art, 2011.

Hanna Arendt, La crise de la culture, Paris, Folio essais, 1954.

Jacques Rancière, Le spectateur émancipé, La fabrique éditions, 2008.

Jean-Paul Colleyn, Le regard documentaire, coll. Supplémentaires, Centre Georges Pompidou, 1993.

Jean-Yves Authier, Marie-Hélène Bacqué, et France Guérin-Pace, Le quartier, enjeux scientifiques, actions politiques et pratiques sociales, Paris : Éd. La découverte, 2007.

John Dewey, L'art comme expérience, Paris, Coll. Folio Essais, Gallimard, 1915. 
Louis Porcher, Les médias entre éducation et communication, Paris, Ed. Vuibert / INA, 2006.

Natacha Cyrulnik, Qu'est-ce que l'éducation artistique au cinéma ?, Ed. L'Entretemps, Coll. Horizons de cinéma, vers les chemins de l'art, 2016.

Philippe Meirieu, Libre parole, réalisé par Jean-Pierre Daniel, DVD-Carnet de route de l'Alhambra Cinémarseille, 2003

Pierre Paillé et Alex Mucchielli, L'analyse qualitative en sciences humaines et sociales, A. Colin, 2005.

Pierre Ricoeur, Parcours de la reconnaissance, Gallimard, Folio Essais, 2004. Jean-Marie Schaeffer, Pourquoi la fiction?, Paris, Seuil, 1999.

Pierre-Michel Menger, Portrait de l'artiste en travailleur - Métamorphoses du capitalisme, Paris, Ed. Le Seuil, 2002

Régis Debray, Manifestes médiologiques, Paris, Gallimard, 1994.

Serge Moscovici (sous la dir.), La psychologie sociale, Quadrige (2e édition) Manuels, PUF, 1984

Serge Tisseron, Enfants sous influence - Les écrans rendent-ils les jeunes violents ? Paris, A. Colin, 2002.

\section{NOTES}

1. Les mobiles évoquent aussi cela

\section{RÉSUMÉS}

Dans le cadre d'ateliers audiovisuels, les réalisateurs Pilar Arcila et Jean- Marc Lamoure ont proposé à des jeunes des quartiers défavorisés de la ville de Port-Saint-Louis-du- Rhône de faire de petits films avec leurs téléphones portables. Alors que ces ateliers de pratiques artistiques sont généralement initiés à l'aide d'une caméra, ce sont les propres téléphones portables des jeunes qui deviennent cette fois l'outil pour réaliser une représentation filmique de leur territoire particulier. Dans ce processus de médiologie (Debray, 1994 ; Bougnoux, 1998), l'outil téléphone permet à la fois de s'emparer d'un objet intime pour construire une nouvelle représentation filmique, du monde, et de soi.

Within the framework of audiovisual workshops, the directors Pilar Arcila and Jean-Marc Lamour proposed to young people of districts disadvantaged by the city of Port- Saint-Louis-du-Rhône to make small movies with their mobile phones. While these workshops of artistic practices are generally introduced by means of a camera, it is the own mobile phones of the young people that become this time the tool to realize a cinematic representation of their particular territory. In this process of médiologie (Debray, 1994 ; Bougnoux, 1998), the tool phones allows at the same time to seize an intimate object to build a new cinematic representation of the world, and one.

En el marco de talleres audiovisuales, los realizadores Pilar Arcila y Jean- Marc Lamoure propusieron a jóvenes de los barrios desfavorecidos por la ciudad de Port-Saint-Louis-du- Rhône 
de hacer pequeñas películas con sus teléfonos móviles. Mientras que estos talleres de practicas artísticas son generalmente iniciados con la ayuda de una cámara, son los proprios teléfonos móviles de los jóvenes que se hacen esta vez la herramienta para realizar una representación cinematográfica de su territorio particular. En esto proceso de médiologie (Debray, 1994 ; Bougnoux, 1998), la herramienta telefonea permite a la vez apoderarse de un objeto intimo para construir une nueva representación cinematográfica, del mundo, y de si.

INDEX

Keywords : mobile phones, mediology, representations, territory, community

Palabras claves : teléfonos móviles, mediología, representaciones, territorio, comunidad

Mots-clés : téléphones portables, médiologie, représentations, territoire, communauté

\section{AUTEUR}

\section{NATACHA CYRULNIK}

Université Aix Marseille, UMR Prism 